\title{
An Alternate Venous Access in COVID-19 Patients Needing Dialysis
}

\author{
Arun Muthukumar
}

\begin{abstract}
The current rapidly rising pandemic scenario due to the SARS COVID-19 infection is known to cause acute respiratory distress syndrome (ARDS) in severely ill patients. Meanwhile, many patients get to suffer multiple comorbidities like septicemia and acute kidney injury (AKI). Most of the critically ill mechanical ventilated patients are nowadays being given trials of prone ventilation for at least one-third duration of a day. These patients may require central venous catheter for various purposes such as fluid resuscitation, vasopressor administration, hemodialysis owing to the fact that many critically ill COVID-19 patients are going for AKI. Central venous access has a major role in accelerating the impending septicemia due to ARDS, by causing catheter-related bloodstream infection, thereby having a synergistic effect in causing sepsis. By using the unconventional methods which are used to give venous access, apart from the regularly used traditional methods of Internal Jugular, subclavian as well as femoral sites, this impending septicemia can be prevented or at least be hampered. This in turn will have major impact in the overall critically ill COVID-19-positive patient's outcome and will have a reduced mortality.

Keywords: Acute kidney injury, Central venous catheter, COVID-19, Catheter-related bloodstream infection, Hemodialysis, translumbar approach. Indian Journal of Critical Care Medicine (2020): 10.5005/jp-journals-10071-23581
\end{abstract}

Sir,

Acute kidney injury (AKI) affects approximately $20-40 \%$ of patients admitted to intensive care units (ICUs) with COVID-19. With 3-9\% population needing renal replacement therapy, early initiation of dialysis has shown improvements in organ prevention. ${ }^{1}$ Dialysis through a femoral or an internal jugular vein has remained the most vital procedures for decades.

According to National Institute of Health COVID-19 Guidelines, a 12-16 hours a day trial of proning has shown positive results in mechanically ventilated patients. ${ }^{2,3}$ COVID patients in the ICU on the other hand require a central vascular access for several purposes: fluid and vasopressors support, parenteral nutrition, hemodynamic monitoring, and repeated blood sampling. Recent studies have also shown that peripherally inserted central line catheter has some advantages over subclavian, femoral or internal jugular veins, while proning and comparatively less catheter site infections. The incidence of AKI brings the need for inserting a proper central venous access. Catheter-related bloodstream infection (CRBSI) also contributes in accelerating the impending sepsis by soiling of the exit site. ${ }^{4}$

One well-studied approach for a long-term venous access is percutaneous translumbar insertion of a central venous catheter into the inferior vena cava. ${ }^{5}$ Studies have elaborated the efficacy of this approach over the traditional in-patients requiring longterm hemodialysis. This method is performed only in patients considered to have few or no other medical options and is not intended as a primary means of central venous access. A metaanalysis observational study of journals showed the average catheter life as 121 days (ranging 14-536 days), with complications in less than $8 \%$ population. ${ }^{4}$ The anticipated complications being site hematoma, thrombosis, tip displacement, and rarely CRBSI. Compared to the traditional methods, translumbar route bypasses some of the factors attributed for CRBSI by overcoming the difficulty like catheter being in a under surface hiding from naked eye, making the routine monitoring and dressings also an easy process.
Department of Anaesthesiology, Calcutta National Medical College, Kolkata, West Bengal, India

Corresponding Author: Arun Muthukumar, Department of Anaesthesiology, Calcutta National Medical College, Kolkata, West Bengal, India, Phone: +91 9445544089, e-mail: arunmuthukumar27@ gmail.com

How to cite this article: Muthukumar A. An Alternate Venous Access in COVID-19 Patients Needing Dialysis. Indian J Crit Care Med 2020;24(9):888-889.

Source of support: Nil

Conflict of interest: None

Nowadays, Tensiocaths, with double lumen 10fr tubes, are used and placed with computed tomography (CT), angio guidance, or fluoroscopy, with or without femoral guidewire. COVID-19 acute respiratory distress syndrome (ARDS) patients requiring prone ventilation for more than three-fourth of a day can be put a CVC by this method, thereby reducing lot of complications and early sepsis. This method has advantages and minimal range of disadvantages like its labor-consuming tendency, and requiring an ongoing CT or running fluoroscopy, at times a radiologist presence, to guide the proper placement of catheter. Practically speaking, this method may be of restricted use in ICUs where portable CTs and fluoroscopies are used. But considering its multiple advantages like dual usage of ports for venous access and dialysis, with a predicable role in preventing morbid complications, this unconventional method may be of importance in the current worsening scenario. One timed insertion of the catheter may serve the purpose of reducing multiple catheter handling and unconscious human errors which compromise the patient health.

So, CT or fluoroscopy-guided emergency translumbar approach may be considered as a good alternate option over the conventional methods, in reducing septicemia in prone-ventilated COVID-19 ARDS patients.

(-) The Author(s). 2020 Open Access This article is distributed under the terms of the Creative Commons Attribution 4.0 International License (https://creativecommons. org/licenses/by-nc/4.0/), which permits unrestricted use, distribution, and non-commercial reproduction in any medium, provided you give appropriate credit to the original author(s) and the source, provide a link to the Creative Commons license, and indicate if changes were made. The Creative Commons Public Domain Dedication waiver (http://creativecommons.org/publicdomain/zero/1.0/) applies to the data made available in this article, unless otherwise stated. 


\section{References}

1. Cheng $Y$, Luo R, Wang $K$, Zhang $M$, Wang Z, Dong L, et al. Kidney disease is associated with in-hospital death of patients with COVID19. Kidney Int 2020;97(5):829-838. DOI: 10.1016/j.kint.2020.03.005.

2. Pan C, Chen L, Lu C, Zhang W, Xia J-A, Sklar MC, et al. Lung recruitability in SARS-CoV-2 associated acute respiratory distress syndrome: a single-center, observational study. Am J Respir Crit Care Med 2020;201(10). DOI: 10.1164/rccm.202003-0527LEAvailable at: https://www.ncbi.nlm.nih.gov/pubmed/32200645.

3. Bamford P, Bentley A, Dean J, Whitmore D, Wilson-Baig N. ICS guidance for prone positioning of the conscious COVID patient.
Intensive Care Soc 2020. Available at: https://emcrit.org/wp-content/ uploads/2020/04/2020-04-12-Guidance-for-conscious-proning.pdf. Accessed May 14, 2020.

4. Grözinger G, Grosse U, Syha R, Hoffmann R, Partovi S, Nikolaou K, et al. CT-guided translumbar placement of permanent catheters in the inferior vena cava: description of the technique with technical success and complications data. Cardiovasc Intervent Radiol 2018;41(9):1356-1362. DOI: 10.1007/s00270-018-1961-9.

5. Kade G, Les J, Buczkowska M, Łabus M, Niemczyk S, Wankowicz Z. Percutaneous translumbar catheterization of the inferior vena cava as an emergency access for hemodialysis - 5 years of experience. J Vasc Access 2014;15(4):306-310. DOI: 10.5301/jva.5000185. 